\title{
Psychometric properties of the Canadian Occupational Performance Measure in home-dwelling older adults
}

This article was published in the following Dove Press journal:

Journal of Multidisciplinary Healthcare

I September 2016

Number of times this article has been viewed

\author{
Hanne Tuntland ${ }^{1,2}$ \\ Mona Kristin Aaslund' \\ Eva Langeland ${ }^{2}$ \\ Birgitte Espehaug ${ }^{3}$ \\ Ingvild Kjeken ${ }^{4,5}$
}

'Department of Global Public Health and Primary Care, Faculty of Medicine and Dentistry, University of Bergen, Bergen, Norway; ${ }^{2}$ Centre for Care Research Western Norway, Bergen University College, Bergen, Norway; ${ }^{3}$ Centre for Evidence-Based Practice, Bergen University College, Bergen, Norway; ${ }^{4}$ National Advisory Unit on Rehabilitation in Rheumatology, Diakonhjemmet Hospital, Bergen, Norway; ${ }^{5}$ Department of Occupational Therapy, Prosthetics and Orthotics, Faculty of Health Sciences, Oslo and Akershus University College of Applied Sciences, Oslo, Norway

Correspondence: Hanne Tuntland Centre for Care Research Western Norway, Bergen University College, PO Box 7030, 5020 Bergen, Norway Tel +4755587839

Email Hanne.Kristin.Tuntland@hib.no
Background: The Canadian Occupational Performance Measure (COPM) is an occupational therapy instrument designed to help participants identify, prioritize, and evaluate performance of important occupations.

Objective: To investigate the validity, responsiveness, interpretability, and feasibility of the COPM when used by various health professions in home-dwelling older adults receiving reablement. Reablement is a new form of multidisciplinary home-based rehabilitation for older adults experiencing functional decline.

Participants and methods: The sample of 225 participants, mean age 80.8 years, who were in need of rehabilitation for various health conditions were included in the study. Data collection was conducted at baseline and at 10 weeks follow-up. The COSMIN guidelines and recommendations for evaluating methodological quality were followed.

Results: Content validity, construct validity, and feasibility were found to be adequate. Responsiveness, however, was moderate. Functional mobility was the most frequently prioritized occupational category of all. Regarding interpretability, the minimal important change was 3.0 points and 3.2 points for performance and satisfaction, respectively. The older adults reported that COPM was a useful and manageable instrument. The majority of the occupational therapists, physiotherapists, and nurses reported that they had the required expertise to conduct the COPM assessments.

Conclusion: The results support the multidisciplinary use of the COPM in clinical practice and research in a home-dwelling, heterogeneous population of older adults. Based on the findings, 3 points are recommended as a cutoff point to distinguish between older adults who have a minimal important change in COPM performance and COPM satisfaction and those who have not. Keywords: rehabilitation, reablement, health services for the aged, COPM, validity, multidisciplinarity

\section{Introduction}

The Canadian Occupational Performance Measure (COPM) is an instrument designed to help participants identify, prioritize, and evaluate occupational performance (COPM-P) and satisfaction with performance (COPM-S) of important occupations they encounter in their daily lives. ${ }^{1}$ The term occupation refers not only to work but also to all kinds of human doing, be it self-care, productivity, or leisure. ${ }^{2}$ The COPM is an occupational therapy tool, which is now being used on a multidisciplinary basis. In Norway, the COPM is widely used in reablement as a tool for goal determination and evaluation. ${ }^{3}$ Also in other countries, COPM is used in reablement. Reablement is a relatively new form of home-based rehabilitation for people experiencing functional decline. The 
intervention, which is time limited, person-centered, and goal directed, is delivered by a multidisciplinary team. ${ }^{4-6}$ In Norway, where rehabilitation is a statutory service in primary care, there has been a rapid implementation of reablement during the last 4 years. ${ }^{7}$ The key question underpinning the emphasis on person-centered practice in reablement is "What are important activities for you now?". ${ }^{8}$ The COPM is used to enhance congruence between participants' needs and priorities, professionals' clinical judgment, intervention priorities, and evaluation of outcomes and is assumed to have the capability to capture the possible effects of reablement. ${ }^{9}$

The psychometric properties of the COPM have been widely tested. In a literature review including 19 methodological studies conducted on various target groups, the authors conclude that the COPM is a valid, reliable (test-retest), responsive, and feasible instrument. ${ }^{10}$ Validity, test-retest reliability, and responsiveness of the COPM have been tested in older adults with various diagnoses, such as stroke, ${ }^{11}$ depression, ${ }^{12}$ hip fracture, ${ }^{13}$ and hand osteoarthritis. ${ }^{14}$ However, only two studies were conducted in a heterogeneous elderly population investigating validity and responsiveness, respectively. ${ }^{15,16}$ Hence, more research on content validity, construct validity, and responsiveness is needed on the oldest of old people. The Norwegian version of the COPM was tested for validity, responsiveness, testretest reliability, and feasibility in people with rheumatic diseases and had good results. ${ }^{14,17}$

Regarding interpretability, that is the degree to which one can assign qualitative meaning to an instrument's quantitative scores or change in scores, ${ }^{18}$ it is stated in the COPM manual that a change of 2 points implies an important change. ${ }^{1}$ However, evidence to support this statement is not confirmed. We find it not plausible that the minimal important change (MIC) is constant, irrespective of diagnoses, severity of disability, age, and the COPM-P versus COPM-S dimensions. Nonetheless, one study has found the optimal cutoff to be 1.37 points and 1.90 points for occupational performance and satisfaction with occupational performance, respectively, but this study was conducted among adults. ${ }^{19}$ As a result, the MIC for the COPM has not yet been evaluated with scientific methods in a population of old people.

Some studies suggest that the COPM assessment may be performed by health professionals other than occupational therapists, ${ }^{20,21}$ but none of the authors have explored the various professions' self-perceived competence in conducting COPM assessments. For this reason, investigation of competence required to conduct COPM assessments, which is considered to be a part of feasibility, is warranted.
Hence, the objective of this study was to investigate the content validity, construct validity, responsiveness, interpretability, and feasibility of the COPM when used by different health professionals in delivering reablement for homedwelling older adults.

\section{Participants and methods Participants}

The sample in the current study was derived from a nationwide, multicenter, clinical controlled trial evaluating the effects of reablement. ${ }^{22}$ The nationwide sample consisted of 833 participants living in 43 different municipalities. The enrollment period lasted from the beginning of April 2014 until the end of June 2015. People applying for, or referred to, public home-based services were potential participants for the study based on their self-reported activity limitations. Some of the participants had been hospitalized due to an acute illness, while others were recruited after having gradually developed functional decline not needing hospitalization or institution-based treatment. People were eligible if they were home dwelling, $>18$ years of age, understood spoken and written Norwegian, and experienced functional decline. The participants were excluded if they were in need of institution-based rehabilitation or nursing home placement or if they were terminally ill or cognitively diminished. The intervention group participated in reablement that lasted for a maximum of 10 weeks. The control group received care as usual.

The first 225 participants, aged 65 years and older, enrolled into the intervention group in the large multicenter study whose data have been collected at baseline and after 10 weeks were included in the current study. Hence, people who had dropped out and people whose data was not registered at 10 weeks follow-up by the time data analysis started were not included.

All participants received information about the study and gave written consent prior to study enrollment. The trial was approved by the Regional Committee for Medical and Health Research Ethics for Western Norway (REK West, 2014/57-1).

\section{Training of data collectors}

As reablement was implemented in 43 different municipalities in the nationwide study, it was essential to train data collectors to ensure high-quality data and complete data sets. A 2-day course was conducted. The first day covered the use of the COPM, containing lectures, demonstrations, and practical exercises. One representative from each of the municipalities attended the course and was responsible for the 
internal training in how to conduct the COPM interview. Each municipality had appointed a contact person to be in charge of communication with the researchers. On the second day, the contact persons were trained in the general procedures of the research project and data collection procedures for the other outcome measures used. Each municipality also received a trial manual that contained information on all the procedures and the data collection instruments. In addition, the researchers had close contact with all municipalities in the course of the data collection and implementation period in order to ensure adherence to the protocol and minimize occurrence of missing data.

\section{Data collection}

We collected demographic characteristic of the sample, including information on health conditions. The participants scored degree of motivation for rehabilitation on a scale from 1 to 10 , where 10 was the best. We used five different outcome measures, all of which were collected at baseline and at treatment conclusion after 10 weeks. The instrument under investigation in the current study was the COPM measuring occupational performance and satisfaction with performance. The other instruments used in the multicentre study were used as comparative instruments in the current study. They comprised physical functioning (measured by the Short Physical Performance Battery [SPPB]), healthrelated quality of life (measured by the European Quality of Life Scale [EQ-5D]), coping (measured by the Sense of Coherence questionnaire [SOC-13]), and positive mental health (measured by the Mental Health Continuum - Short Form [MHC-SF]). Data were collected by the clinicians in the reablement teams who also delivered the interventions. The clinicians were blinded for the assessment when reassessing the COPM.

The COPM is a patient-specific measure, which means that it is focusing on issues that matter to each participant. ${ }^{23}$ The instrument measures a person's self-perceived occupational performance within three occupational performance areas. Occupational performance is perceived as the result of interaction and interdependence between the person(s), the environment, and the occupation(s). ${ }^{1}$ We used the Norwegian translation of the fourth edition of the instrument. ${ }^{24}$ During a semistructured interview, the participants described which occupations they considered were important but difficult to perform. The importance of each occupation was thereafter rated on a $1-10$-point scale $(10=$ very important $)$. Next, the participants prioritized a maximum of five of the most important occupations and rated performance and satisfaction with performance for each of these occupations on a scale from 1 to 10 (a higher score reflected better performance or higher satisfaction). Sum scores for the COPM-P or COPM-S, respectively, were calculated by adding the performance or satisfaction scores and thereafter dividing by the number of prioritized occupations.

After the COPM interview was finished, the participants were asked to rate to what degree they felt that the COPM was useful in determining goals for rehabilitation (scale 1-10, 10= very useful), and they answered open questions regarding the scoring process, their experiences, and possible difficulties in completing the interview and scoring. Furthermore, the health care providers conducting the COPM interviews were asked to what degree they considered that the results from the assessment were useful as a basis for planning and evaluating the intervention and to rate the difficulty they experienced assisting the participant during the interview (scale 1-10, 10= very useful or very simple). Their education and need for further education in the COPM were also recorded.

The SPPB is a screening test for mobility and aims at identifying people at risk of functional decline. ${ }^{25}$ The test includes a balance test, a gait test, and a chair stand test. The gait test involves $4 \mathrm{~m}$ of walking in preferred walking speed. Good validity, reliability, and responsiveness have been reported in a systematic review using studies where community-dwelling older adults were investigated. ${ }^{26}$

EQ-5D measures health-related quality of life. The instrument consists of two parts, a questionnaire and a visual analog scale (VAS). The questionnaire has five domains (mobility, self-care, usual activities, pain/discomfort, and anxiety/depression) with five levels (no problems to extreme problems). The VAS gives an indication of how the participants assess their own health on a $0-100$ scale, with 100 being excellent health. A structured review of the psychometric properties of the EQ-5D concluded that there is good evidence for reliability, validity, and responsiveness among older adults. ${ }^{27}$

Coping was measured by the SOC-13, which was developed by Antonovsky. ${ }^{28}$ The self-reported questionnaire comprised 13 items. A systematic review of 127 studies with samples of various diagnosis and age groups concluded that the SOC scale is a reliable, valid, and cross-culturally applicable instrument measuring how people manage stress and stay well. ${ }^{29}$

Positive mental health was measured by the MHC-SF. This instrument measures three dimensions of the positive mental health concept. ${ }^{30}$ Each of the 14 items is scored by rating the frequency of various feelings during the past month 
on a 6-point scale from never (0) to every day (5). Higher scores imply higher levels of positive mental health. Validity and reliability have been shown to be good in a study with a large sample of people aged $18-87$ years. $^{31}$

A 5-point global rating scale question was used to capture the participants' impression of change at 10 weeks follow-up. The question was: "To what degree have you experienced a change in management in daily activities since the start of reablement 10 weeks ago"? The five responses were: 1) "much improved"; 2) "a little improved"; 3) "no change"; 4) "a little deteriorated"; and 5) "much deteriorated".

The municipalities selected which professionals should administer which instruments. Usually, one participant was evaluated by one or two professionals who administered all the instruments.

\section{Data analysis}

The COSMIN guidelines and recommendations for evaluating methodological quality were followed. ${ }^{32,33}$ The acronym COSMIN stands for COnsensus-based Standards for the selection of health Measurement INstruments. The COSMIN guidelines are based on international consensus on taxonomy, terminology, and definitions of measurement properties for health-related patient-reported outcomes. ${ }^{18}$

\section{Content validity}

According to the COSMIN terminology, content validity is defined as the degree to which the content of an instrument is an adequate reflection of the construct measured. ${ }^{33}$ The constructs in question in the COPM are occupational performance and satisfaction with performance. Content validity was explored by answering four relevant questions: ${ }^{32,33} 1$ ) Do all occupational categories in the COPM refer to relevant aspects of the construct? 2) Are all occupational categories relevant for the study population, for example with regard to sex and age? 3) Are all occupational categories relevant for the purpose of the instrument? and 4) Do all occupational categories together comprehensively reflect the construct?

The first question was addressed by examining whether the identified occupations in fact were occupational categories covering relevant aspects of the construct. The second question was addressed by exploring the prioritized occupations listed by the participants. Issues of interest concerning the study population were distribution of prioritized occupations according to sex and whether the occupations were relevant for older adults. The third question was answered by examining whether occupations were described within all relevant occupational categories and whether the participants were able to score the identified occupations for performance and satisfaction with performance on a 10-point scale at baseline and follow-up. The fourth question was addressed by asking each participant after the COPM interview and scoring was completed whether he or she had other important occupations not covered in the COPM interview. The participants' narrative answers were written down, grouped, and categorized.

Content validity was regarded as confirmed if $>80 \%$ of the participants' answers confirmed questions 1, 3, and 4. Since some of the occupational categories are age specific, it cannot be expected that all occupations will be equally relevant for old persons as for young ones (question 2). The principal investigator performed the analysis of the participants' answers.

\section{Construct validity}

Construct validity was defined as the degree to which the COPM scores were consistent with hypotheses stating that the instrument in question validly measures the construct to be measured. ${ }^{33}$ We developed hypotheses covering all comparative instruments and both the two outcomes COPM-P and COPM-S. Hence, construct validity was based on a priori hypotheses for levels of correlation between baseline COPM-P and COPM-S sum scores and sum scores for mobility (SPPB), scores for the gait test (part of the SPPB), single-item scores on usual activities (EQ-5D), VAS scores of health-related quality of life, and sum scores for coping (SOC-13) and mental health (MHC-SF). When the instruments were measuring different constructs, low correlations were expected. Even when the constructs were similar, only low (to moderate) correlations were expected since the COPM is a patient-specific and not a fixed-item instrument, whereas the other instruments are performance tests or questionnaires with standardized items. We expected the COPM to correlate higher with the EQ-5D VAS score, and in particular with EQ-5D usual activities and the SPPB gait test, based on an assumption that these items corresponded most with the construct of occupational performance (Table 1).

\section{Responsiveness}

Responsiveness was defined as the ability of the COPM instrument to detect change over time in the construct measured. ${ }^{18}$ Evaluation of responsiveness was based on testing a priori hypotheses regarding mean differences of change scores for COPM-P and COPM-S compared with various global rating scale responses (Table 2). Our hypotheses postulated large differences in mean COPM change scores 
Table I Construct validity hypotheses and results

\begin{tabular}{llllll}
\hline Instrument & Dimension & COPM dimension & Hypotheses $^{\mathbf{a}}$ & Results & Confirmed hypotheses (yes/no) \\
\hline SPPB & Sum score physical function & Performance & Low & $0.22^{*}$ & Yes \\
SPPB & Single-item score, gait test & Performance & Low/moderate & 0.13 & Yes \\
EQ-5D & Single-item score, usual activities & Performance & Low/moderate & $-0.36^{*}$ & Yes \\
EQ-5D & VAS score health today & Performance & Low/moderate & $0.23^{*}$ & Yes \\
SOC-I3 & Sum score coping & Performance & Low & 0.02 & Yes \\
SOC-13 & Sum score coping & Satisfaction & Low & 0.04 & Yes \\
MHC-SF & Sum score mental health & Performance & Low & 0.03 & Yes \\
MHC-SF & Sum score mental health & Satisfaction & Low & -0.02 & Yes \\
\hline
\end{tabular}

Notes: axpected level of Spearman's correlations. *Correlation is significant at 0.01 level (two-tailed).

Abbreviations: COPM, Canadian Occupational Performance Measure; SPPB, Short Physical Performance Battery; EQ-5D, European Quality of Life Scale; VAS, visual analog scale; SOC-13, Sense of Coherence questionnaire; MHC-SF, Mental Health Continuum - Short Form.

Table 2 Responsiveness hypotheses and results

\begin{tabular}{|c|c|c|c|}
\hline Instrument & Hypotheses & Result & Confirmed hypotheses (yes/no) \\
\hline Global rating scale & $\begin{array}{l}\text { Significant mean difference in COPM-P change score for } \\
\text { "no change" versus "a little improved" }\end{array}$ & $-1.45^{*}$ & Yes \\
\hline Global rating scale & $\begin{array}{l}\text { Significant difference in mean COPM-S change score for } \\
\text { "no change" versus "a little improved" }\end{array}$ & -1.12 & No \\
\hline Global rating scale & $\begin{array}{l}\text { Significant difference in mean COPM-P change score for } \\
\text { "a little improved" versus "much improved" }\end{array}$ & $-1.53 * *$ & Yes \\
\hline Global rating scale & $\begin{array}{l}\text { Significant difference in mean COPM-S change score for } \\
\text { "a little improved" versus "much improved" }\end{array}$ & $-1.61 * *$ & Yes \\
\hline SPPB (sum score) & $\begin{array}{l}\text { Low correlation between SPPB change scores and } \\
\text { COPM-P change scores }\end{array}$ & $0.40 * *$ & No \\
\hline EQ-5D (single-item score) & $\begin{array}{l}\text { Low correlation }{ }^{\mathrm{b}} \text { between EQ-5D self-care change scores } \\
\text { and COPM-P change scores }\end{array}$ & $-0.33^{* *}$ & No \\
\hline SOC-13 (sum score) & $\begin{array}{l}\text { Low correlation }{ }^{\mathrm{b}} \text { between SOC- } 13 \text { change scores and } \\
\text { COPM-P change scores }\end{array}$ & 0.11 & Yes \\
\hline MHC-SF (sum score) & $\begin{array}{l}\text { Low correlation }{ }^{b} \text { between MHC-SF change scores and } \\
\text { COPM-P change scores }\end{array}$ & 0.17 & Yes \\
\hline
\end{tabular}

Notes: andependent samples $t$-test. 'Pearson's correlation coefficient. *Statistically significant at 0.05 level (two-tailed). **Statistically significant at $0.0 \mathrm{I}$ level (two-tailed). Abbreviations: COPM, Canadian Occupational Performance Measure; COPM-P, COPM measuring occupational performance; COPM-S, COPM measuring satisfaction with performance; SPPB, Short Physical Performance Battery; EQ-5D, European Quality of Life Scale; SOC-I3, Sense of Coherence questionnaire; MHC-SF, Mental Health Continuum - Short Form.

between groups defined by responses on the global rating scale ("no change" versus "a little improved"; "a little improved" versus "much improved"). In line with the COSMIN guidelines, evaluation of responsiveness was also based on testing predefined hypotheses for levels of correlation for change values (differences between 10 weeks follow-up and baseline scores between the COPM-P scores and scores for physical function [SPPB], single-item scores on self-care [EQ-5D], coping [SOC-13], and mental health [MHC-SF]).

As the intervention focused on occupational performance, we expected moderate-to-large changes in COPM-P, whereas we expected no or small changes in mobility measured with the SPPB, mental health measured with the MHC-SF, or coping measured with SOC-13. Thus, we hypothesized low correlations between COPM-P change scores and the change scores of these comparative instruments. Furthermore, even if self-care (measured by the EQ-5D) and COPM-P cover the same construct (occupational performance), we did not expect that the single EQ-5D item would capture change in self-care following reablement, as the self-care item implies only personal hygiene and dressing, while the COPM construct implies all kinds of daily activities. We therefore hypothesized low correlation here as well.

\section{Interpretability}

According to the COSMIN terminology, interpretability is the degree to which one can assign qualitative meaning to an instrument's quantitative scores or change in scores. ${ }^{18} \mathrm{In}$ the current study, the important aspect was to determine the size of the MIC, which is defined as the smallest change in score which individual participants perceive as important. ${ }^{32}$ We used an anchor-based approach to determine the MIC. The 5-point global rating scale was used as a gold standard to capture the participants' impression of change in coping 
with their daily activities at 10 weeks follow-up. The global rating scale is suitable provided the change question refers to the same construct as the instrument under study. ${ }^{32}$ Owing to few participants, the responses in the categories "a little deteriorated" and "much deteriorated" are not reported.

\section{Feasibility}

Even if feasibility is not a psychometric property, it is described in the COSMIN recommendations as a relevant issue to consider when assessing outcome measures. ${ }^{32}$ Feasibility in this type of study refers to whether participants are able to answer the questions in the instrument and may be evaluated by exploring response rate, time spent on completion of the COPM data collection, patient burden, and required clinical expertise. We recorded the response rate and calculated median time spent on the COPM interview at baseline and follow-up. The participant's narrative answers were written down. Thereafter, the principal investigator grouped and categorized the data.

Five different health professionals were involved in the COPM assessments (nurses, occupational therapists, physiotherapists, auxiliary nurses, and social educators). However, since some interviews were performed by two or three different professionals together, these assessments were excluded from the analysis $(n=39)$ in order to be able to compare the professionals separately. Moreover, since there was only one social educator and five auxiliary nurses, their assessments were excluded when performing significance tests.

\section{Statistical analysis}

Demographic characteristics of the participants and the COPM baseline scores were described by frequencies and mean/median scores.

\section{Construct validity and responsiveness}

Correlation tests of hypotheses are an established method of confirming construct validity and responsiveness. ${ }^{32}$ Depending on the distribution of the scores, Spearman's rho correlation or Pearson's $r$ was used for testing hypotheses. A high correlation was defined as $r \geq 0.60$, moderate correlation as $r>0.30$ and $r<0.60$, and a low correlation as $r \leq 0.30 .{ }^{34}$ Hypotheses of mean differences in COPM change scores comparing participants with various global rating scale responses ("no change" versus "a little improved", "a little improved" versus "much improved") were tested with independent samples $t$-tests. In accordance with Terwee et al, ${ }^{35}$ adequate construct validity and responsiveness were established when $>75 \%$ of the hypotheses were confirmed.

\section{Interpretability}

Differences in mean change scores of COPM-P and COPM-S between the five different categories in the global rating scale were determined by independent samples $t$-tests. The change score in the category "a little improved" was considered to reflect the MIC.

\section{Feasibility}

Differences in self-perceived experience and competence between health professionals (nurses, occupational therapists, physiotherapists) conducting the COPM interviews were examined statistically with chi-square tests for categorical variables and one-way analysis of variance (F-tests) for continuous variables.

For the statistical analysis, IBM SPSS Statistics Version 22 (IBM Corporation, Armonk, NY, USA) was used. All $P$-values $<0.05$ were considered to be statistically significant.

\section{Results}

\section{Participants}

The 225 participants were primarily female $(72 \%)$, the mean age was 80.8 years, and $76 \%$ lived alone. They had a variety of reasons for needing rehabilitation, most frequently fractures and balance problems, and they had a median of three additional health conditions. The participants included in this study lived in 32 of the 43 possible municipalities, representing 16 out of 19 counties stretching out from the south to the north of Norway. A total of $13.6 \%$ of the sample in the multicenter study dropped out at 10 weeks follow-up. However, an analysis comparing the participants who completed the study and the participants who dropped out showed no significant differences in baseline COPM-P and COPM-S scores ( $P=0.87$ and $P=0.83$, respectively). Table 3 presents the baseline demographic characteristics.

\section{Health care providers}

A total of 78 health care providers conducted the assessments of the 225 participants. There were 12 nurses, 33 occupational therapists, 27 physiotherapists, five auxiliary nurses, and one social educator.

\section{Content validity}

The first question to be answered dealt with whether all occupational categories in the COPM refer to relevant aspects of the construct. Figure 1 shows the distribution of prioritized occupations in total and for each sex. The participants described a total of 1,371 occupations and prioritized 757 of these. The occupational performance areas 
Table 3 Baseline characteristics

\begin{tabular}{|c|c|}
\hline \multicolumn{2}{|l|}{ Baseline characteristics $(\mathrm{N}=\mathbf{2 2 5})$} \\
\hline Age, mean years (SD), range & $80.8(6.7), 65-95$ \\
\hline Female, n (\%) & $162(72.0)$ \\
\hline Living alone, $\mathrm{n}(\%)$ & I7I (76.0) \\
\hline Higher education $\geq$ university/university college, $n(\%)$ & $48(21.3)$ \\
\hline Retired, $n(\%), n=221$ & $219(97.3)$ \\
\hline $\begin{array}{l}\text { Motivation for rehabilitation, scale } 1-10,10 \text { is best, } \\
\text { mean (SD), } n=223\end{array}$ & $8.17(2.0)$ \\
\hline $\begin{array}{l}\text { Occupational performance (COPM-P) sum score, } \\
\text { mean (SD), } n=223\end{array}$ & $3.52(1.7)$ \\
\hline $\begin{array}{l}\text { Occupational satisfaction (COPM-S) sum score, mean } \\
\text { (SD), } n=223\end{array}$ & $3.38(1.7)$ \\
\hline \multicolumn{2}{|l|}{ Major health condition, n (\%) } \\
\hline Fracture & $53(23.6)$ \\
\hline Dizziness/balance problem & $40(17.8$ \\
\hline Pain & $24(10.7)$ \\
\hline Stroke & $18(8.0)$ \\
\hline Cardiovascular disease & $15(6.7)$ \\
\hline Problem/disease in back, hip, knee, or ankle & $14(6.2)$ \\
\hline Rheumatoid arthritis/arthrosis & II (4.9) \\
\hline Respiratory disease & $10(4.4)$ \\
\hline Unspecified functional decline & $7(3.1)^{\prime}$ \\
\hline Vision problem/eye disease & $4(1.8)$ \\
\hline Cancer & $4(1.8)$ \\
\hline Mental illness & $4(1.8)$ \\
\hline Other health condition & $21(9.3)$ \\
\hline $\begin{array}{l}\text { Number of additional health conditions, median } \\
\text { (SD), IQR }\end{array}$ & $3(3.0), 0-9$ \\
\hline
\end{tabular}

Note: $\mathrm{n}$ is specified in the table only when the amount of participants was $<225$. Abbreviations: COPM, Canadian Occupational Performance Measure; COPM-P, COPM measuring occupational performance; COPM-S, COPM measuring satisfaction with performance; IQR, interquartile range; SD, standard deviation.

with $>70$ responses are specified in order to illustrate the most frequently prioritized occupations (Figure 1). Fiftyfour statements $(7 \%)$ could not be categorized into any of the occupational categories of the COPM. Forty-nine of these statements were body function items according to the International Classification of Functioning, Disability and Health terminology, ${ }^{36}$ such as balance, strength, endurance, memory, and pain, while the remaining five occupations were to prevent falls, to have regular meals, to have extra energy, to have motivation for outdoor walks, and to remove plaster. To conclude, only $7 \%$ of the items/occupations did not refer to the construct.

The second question to be answered was whether all occupational categories were relevant for the study population, for example with regard to sex and age. Of the 757 prioritized occupations, $67 \%$ were in the self-care domain, $20 \%$ were in the leisure domain, and $13 \%$ were in the productivity domain. The most dominating occupational category was functional mobility ( $40 \%$; Figure 1). As could be expected in a retired population, paid/unpaid work $(0.3 \%)$ and play/school $(0 \%)$ were prioritized by only a few participants. Relative to their proportion, household management, socialization, and quiet recreation appear to be more frequently prioritized occupations for females than for males in this population of older adults. To summarize, the frequency of prioritized occupations varied among the nine occupational categories and there were also sex-specific and age-specific variations.

The third question concerned whether all occupational categories were relevant for the purpose of the instrument. Almost all participants ( $>99 \%$ ) were able to define occupations, thereby confirming that the instrument served the purpose of goal determination in reablement of elderly people. Likewise, almost all participants (>99\%) were able to rate performance and satisfaction with performance at baseline and follow-up, which demonstrates that the instrument's purpose of evaluation was also satisfactory (the number of missing scores was 2 and 0 for COPM-P and 1 and 2 for COPM-S at baseline and 10 weeks followup, respectively).

Finally, the fourth question was whether all occupational categories together comprehensively reflect the construct. Following the COPM interview, 27 participants (12\%) reported a total of 29 important occupations and items not covered in the interview ( 20 occupations reported by females and nine occupations by males). Almost all of these could, however, be categorized into the following occupational categories or items: active recreation $(n=10)$, quiet recreation $(n=6)$, socialization $(n=4)$, functional mobility $(n=2)$, community management $(n=2)$, personal care $(n=1)$, household management $(n=1)$, body function (sleep and hearing; $n=2$ ), and unclassifiable (to be independent; $n=1$ ). This categorization was performed retrospectively based on the clinical judgment of the principal investigator. The results demonstrate that occupations not identified during the COPM interview also reflected the construct.

\section{Construct validity}

As shown in Table 1, all the hypotheses were confirmed, demonstrating that the construct validity of the COPM is adequate. The findings show that, in general, there is a low correlation between the COPM and the other instruments. We found a moderate correlation between COPM-P and EQ-5D usual activities, indicating that these two indices partly measure the same construct.

\section{Responsiveness}

The mean difference between COPM change scores among those answering "no change" versus "a little improved" and 


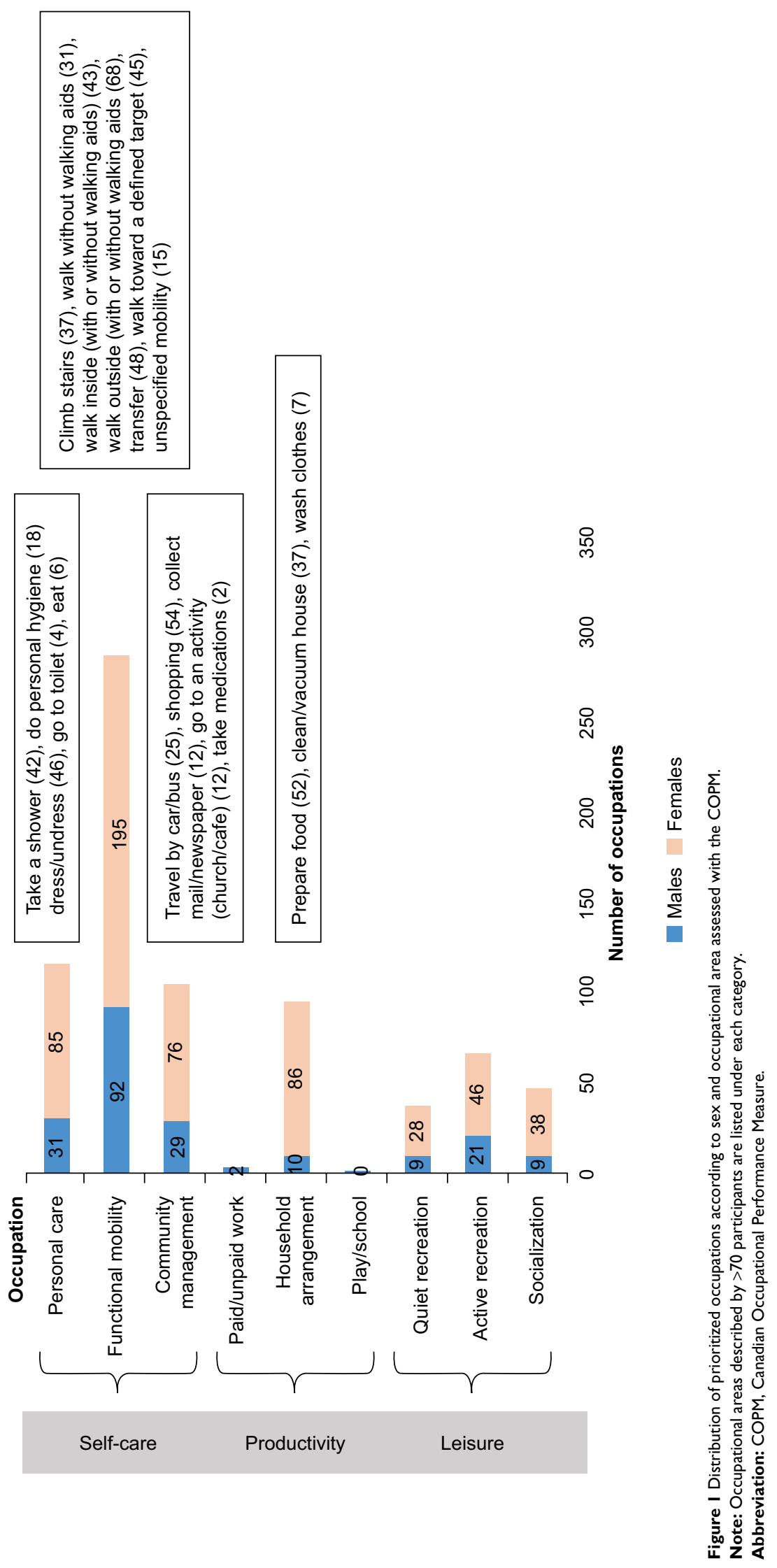


Table 4 The mean change scores (SD) for occupational performance and satisfaction with performance scored on a numerical rating scale (range $1-10$ ), according to participants' answers in the global rating scale of perceived change

\begin{tabular}{lll}
\hline $\begin{array}{l}\text { Global perceived } \\
\text { change }\end{array}$ & $\begin{array}{l}\text { Number of } \\
\text { participants }\end{array}$ & $\begin{array}{l}\text { Mean change } \\
\text { score (SD) }\end{array}$ \\
\hline $\begin{array}{l}\text { COPM-P } \\
\text { Much improved }\end{array}$ & 97 & $4.6(2.1)$ \\
A little improved & 74 & $3.0^{*}(2.0)$ \\
$\quad$ No change & 28 & $1.6(2.2)$ \\
COPM-S & & \\
$\quad$ Much improved & 96 & $4.8(2.1)$ \\
A little improved & 73 & $3.2^{*}(2.1)$ \\
No change & 28 & $2.2(2.2)$ \\
\hline
\end{tabular}

Notes: Independent samples $t$-tests performed. *The MIC.

Abbreviations: COPM, Canadian Occupational Performance Measure; COPM-P, COPM measuring occupational performance; COPM-S, COPM measuring satisfaction with performance; MIC, minimal important change; SD, standard deviation.

"a little improved" versus "much improved" was statistically significant for three of the four tested hypotheses. The correlations between difference in COPM-P change scores and the comparative instruments' change scores were low to moderate. As such only two of the four correlation hypotheses were confirmed. Table 2 shows that $62.5 \%$ (five of eight) of the hypotheses were confirmed, indicating that the responsiveness of the COPM in this population is moderate.

\section{Interpretability}

As shown in Table 4, the MIC (mean change score in participants who reported "a little improved" after 10 weeks) is 3.0 points and 3.2 points for COPM-P and COPM-S, respectively.

\section{Feasibility}

The COPM response rate was $99.5 \%$ and $100 \%$ for COPM-P and $99.5 \%$ and $99.1 \%$ for COPM-S at baseline and followup, respectively. The median time values (range) for the COPM at baseline and follow-up interview were 33 minutes (10-100 minutes) and 9 minutes (2-68 minutes), respectively.

The participants' mean (SD) rating of the degree to which they felt that the instrument was useful for goal determination was 7.8 (2.0). When examining the participants' narrative answers $(n=225)$, the majority $(82 \%)$ of the participants stated that they experienced the interview and scoring as useful. In particular, they experienced that the COPM interview contributed to information about "what is important to me". Furthermore, they felt that the COPM interview led to a greater awareness about their daily lives and to a feeling of being seen and listened to. They also described that the interview enhanced their motivation to focus on improving occupational performance, and that information brought forward during the interview and scoring process was useful as a basis for developing rehabilitation goals. However, $\sim 10 \%$ of the participants also described difficulties with answering questions and scoring or regarded the instrument as less useful in the goal-setting process. A deeper exploration of the perceived difficulties revealed that these participants mainly experienced difficulties related to scoring; however, these difficulties were less at follow-up. In addition, some participants regarded defining a score as very abstract or theoretical and explained that they were not accustomed to thinking in this way. Finally, some participants (8\%) did not give any explanation for their responses.

Concerning the question of addressing participants' experiences related to describing occupations and defining occupational goals, the majority of the participants (89\%) regarded these as positive. They answered that it was "okay", referring to the interview situation, and "easy" referring to the process of goal determination. However, almost $9 \%$ of the participants also described negative experiences, most frequently related to difficulties with identifying occupations and defining goals. A minority of them said that they felt the interview itself was tiring and time consuming. Finally, some participants $(2 \%)$ did not give any explanation for their negative experiences.

The health care providers' mean (SD) score of the degree to which they considered that the results from the assessment were useful as a basis for planning and evaluating on the intervention was 8.2 (1.7; Table 5). Moreover, they described that they experienced a few difficulties, 7.5 (2.0), when assisting the participant during the baseline COPM interview. Most of the health care providers had taken courses $(61.8 \%) \mathrm{and} /$ or other education in the use of the COPM $(66.2 \%)$. However, $29.0 \%$ of them felt a need for additional COPM education, thereby indicating that they regarded their expertise as insufficient.

The occupational therapists, physiotherapists, and nurses believed that the COPM was useful, that they had few difficulties conducting the COPM interview, and that they had sufficient formal competence in the COPM assessment but needed to some degree additional education (Table 5). However, the one profession that was without a bachelor-level qualification deviated the most from this pattern, namely, the auxiliary nurses. These professionals considered the COPM least suitable for planning and evaluation, demonstrated less competence, and wanted more education in the COPM assessment. 
Table 5 Various health care professionals' self-perceived experience and competence when conducting the COPM baseline interview

\begin{tabular}{|c|c|c|c|c|c|c|c|}
\hline Characteristics & $\begin{array}{l}\text { Total } \\
(n=78)\end{array}$ & $\begin{array}{l}\text { Nurses } \\
(n=12)\end{array}$ & $\begin{array}{l}\text { Occupational } \\
\text { therapists }(n=33)\end{array}$ & $\begin{array}{l}\text { Physiotherapists } \\
(n=27)\end{array}$ & $P$-value & $\begin{array}{l}\text { Auxiliary } \\
\text { nurses }(n=5)\end{array}$ & $\begin{array}{l}\text { Social } \\
\text { educator }(n=1)\end{array}$ \\
\hline $\begin{array}{l}\text { Number of COPM interviews per } \\
\text { professional, mean (SD), } n=186\end{array}$ & $3.3(3.4)$ & I.I (0.3) & $4.1(4.0)$ & $2.7(2.5)$ & 0.001 & I.89 (0.9) & $\mathrm{I} .0$ (NA) \\
\hline $\begin{array}{l}\text { Degree of COPM goals considered } \\
\text { useful for planning and evaluation by } \\
\text { health care provider, baseline, scale } \\
1-10,10 \text { is the best, mean (SD), } n=180^{a}\end{array}$ & $8.2(1.7)$ & $7.9(1.2)$ & $8.4(1.4)$ & $8.2(1.5)$ & 0.3 & $4.4(3.5)$ & 9.0 (NA) \\
\hline $\begin{array}{l}\text { Degree of perceived difficulty assisting } \\
\text { the participant during the baseline } \\
\text { COPM interview, scale } 1-10,10 \text { is the } \\
\text { most simple, mean (SD), } n=180^{a}\end{array}$ & $7.5(2.0)$ & $7.3(2.2)$ & $7.7(1.8)$ & $7.3(2.4)$ & 0.4 & $6.8(1.9)$ & 6.0 (NA) \\
\hline $\begin{array}{l}\text { COPM assessor has taken COPM } \\
\text { course, } n(\%) \text { "yes", } n=170^{a}\end{array}$ & $105(6 \mid .8)$ & $10(58.8)$ & 7I (72.4) & $23(47.9)$ & 0.01 & 0 & I $(100)$ \\
\hline $\begin{array}{l}\text { Number of course days in COPM, } \\
\text { mean (SD), } n=176^{\mathrm{a}}\end{array}$ & $2.0(0.9)$ & I.3 (0.4) & $2.2(0.9)$ & $1.6(1.0)$ & 0.003 & 0 & $\mathrm{I} .0$ (NA) \\
\hline $\begin{array}{l}\text { Other/additional COPM education } \\
\text { taken, } n(\%) \text { "yes", } n=145^{\mathrm{a}}\end{array}$ & $96(66.2)$ & II (100) & $47(58)$ & $30(66.7)$ & 0.02 & $8(100)$ & 0 \\
\hline $\begin{array}{l}\text { Self-perceived need for additional } \\
\text { COPM education, } n(\%) \text { "yes", } n=177^{\mathrm{a}}\end{array}$ & $51(29.0)$ & $8(44.4)$ & $22(21.6)$ & $14(29.2)$ & 0.1 & $7(87.5)$ & I $(100)$ \\
\hline
\end{tabular}

Note: a Each health care provider has performed several COPM assessments.

Abbreviations: COPM, Canadian Occupational Performance Measure; NA, not applicable; SD, standard deviation.

\section{Discussion}

This study supports the use of the COPM in an elderly home-dwelling population and its application as an outcome measure within reablement. The study focused on the validity, responsiveness, interpretability, and feasibility of COPM in a heterogeneous population of 225 home-dwelling older adults. In general, the results show adequate content and construct validity and suggest moderate responsiveness to change. Functional mobility was the most frequently prioritized occupational category of all. The MIC was found to be 3.0 points and 3.2 points for COPM-P and COPM-S, respectively. The majority of the occupational therapists, physiotherapists, and nurses reported that they had the required expertise to conduct the COPM assessments. Having a bachelor-level qualification as a health care provider seems to be an advantage when conducting the COPM assessments.

\section{Content validity}

The sex pattern related to prioritized occupations and the low proportion of participants reporting problems related to paid/unpaid work and play/school were in line with what would be expected in this population of old and retired participants, thereby confirming content validity. Furthermore, the finding that functional mobility was the most frequently prioritized occupation by the participants is in accordance with the results from other studies on older adults. ${ }^{9} 13,16,20,37$ This suggests that mobility is a key priority among older adults as a basis for management of self-care, productivity, and leisure occupations and underlines that mobility is important to address in interventions aimed at enhancing occupational performance and satisfaction with performance in this age group.

\section{Responsiveness}

In this study, $<75 \%$ of the responsiveness hypotheses were confirmed. However, according to de Vet et al, ${ }^{32}$ responsiveness can be considered to be high when $<25 \%$ of the hypotheses are rejected, moderate if $25 \%-50 \%$ of the hypotheses are rejected, and poor when $>50 \%$ of the hypotheses are rejected. In our study, three out of eight (37.5\%) of the responsiveness hypotheses were rejected, suggesting moderate responsiveness. These results are in contrast to the other responsiveness study on a heterogeneous old population, where high responsiveness was indicated. ${ }^{16}$ However, in this study, high responsiveness was not determined by testing hypotheses, but simply by stating that $73 \%$ of the participants reported a change score of $\geq 2$ points. Thus, the methodology used in the two studies differs.

Owing to a few participants in the present study in the groups reporting "no change" in performing their daily activities at 10 weeks follow-up, the power to detect statistically significant mean differences between these participants and those who reported "a little improved" may, however, be questioned. Furthermore, as correlations usually are lower when assessing change scores than 
single scores, low correlations were expected. In hindsight, we acknowledge that we underestimated EQ-5D's responsiveness and therefore should have hypothesized a higher correlation between change scores in COPM-P and EQ-5D self-care since they both measure the construct of occupation. In addition, we did not expect the SPPB sum change score to correlate moderately with the COPM-P change score, given the SPPB sum score includes not only gait but also balance and chair stand. However, this might be explained by the fact that the majority of the prioritized occupations were functional mobility.

As discussed in other studies adhering to the COSMIN recommendations, it is difficult to develop hypotheses concerning correlations between change scores measured with different instruments, since such hypotheses are mostly based on clinical experience. ${ }^{38,39}$ It is even more difficult when a patient-specific instrument is involved, as instruments such as the COPM may capture a wide variety of occupations, which thereafter are compared to instruments with a more limited number of fixed occupational domains. ${ }^{21}$ Thus, it is hard to predict which occupations will be chosen and prioritized by the older adults during the COPM interview. This illustrates that there is a random factor involved, which under other circumstances, might have resulted in $75 \%$ of the hypotheses being confirmed.

\section{Interpretability}

The MIC was calculated to be 3.0 points and 3.2 points for COPM-P and COPM-S, respectively, which is above the suggested MIC of 2 points in the COPM manual. ${ }^{1}$ In general, the MIC probably varies among diagnoses and age groups and therefore needs to be determined according to specific patient groups. The clinical implication of the results is that larger improvements in performance and satisfaction than previously recommended are needed if older individuals receiving reablement perceive an improvement as important. Another implication is that at least 3 points should be considered as a cutoff point in order to distinguish between older adults who report a clinically important change and those who do not.

\section{Feasibility}

The median time for the baseline COPM interview of $33 \mathrm{~min}-$ utes was in accordance with the time frame of $30-40$ minutes in studies on adults and early older adults. ${ }^{14,40}$ The relatively moderate time use was therefore less than expected in this elderly and frail population, based on a general perception that older adults need more time to complete a task.
Furthermore, the majority of the participants felt that the instrument was useful and reported that their experiences with the instrument were positive. Moreover, almost all the participants were able to complete the COPM interview. Consequently, the COPM is a useful and manageable instrument in a population of older adults.

Some participants experienced problems related to the scoring system. Difficulties with numeric scoring procedures in the COPM are also common for younger adults. ${ }^{17,19,40,41} \mathrm{As}$ such, it might not be a generational issue, but a general difficulty for participants of all ages. Hence, in line with Kjeken et al, ${ }^{17}$ the results of this study do not support the hypotheses that scoring problems increase with older age. However, in general, clinicians might need to develop strategies for overcoming the problems with the scoring procedures.

The occupational therapists felt most competent performing the COPM assessments. This is no surprise as occupation is the core domain of concern in occupational therapy practice and education. ${ }^{2}$ The reasons why the auxiliary nurses to a lesser degree thought that the COPM goals were suitable for planning and evaluation were presumably caused by a lack of comprehensive understanding of the instrument's purpose, nature, or conceptual basis. Consequently, having a bachelorlevel qualification in health care seems to be an advantage when conducting COPM assessments. These results should, however, be interpreted with caution, since the number of participants in some of the groups was small.

At any rate, the clinical implications of these results might be to underpin the COPM training when used in a multidisciplinary context, as argued by Enemark and Carlsson. ${ }^{20}$

\section{Strengths and limitations}

A strength of this study is that there were few missing data. Another strength is the diagnostic and geographical heterogeneity among the participants, which implies that the results may be generalizable within this age group. However, in this study, we have examined a generic population of older adults and a generic intervention to establish the psychometric properties of the COPM. Hence, the results we found may not be generalizable to specific diagnostic groups and specific interventions, even in an elderly population. This refers in particular to the responsiveness and the MIC.

A limitation of this study is that significance testing of experience and competence among all health professionals could not be performed, due to a small number of auxiliary nurses and social educators. In addition, although a dropout analysis was performed at 10 weeks follow-up, selection bias cannot be ruled out. Furthermore, test-retest reliability of 
the COPM is not established in this population and should be investigated in future studies. Likewise, responsiveness should also be retested.

\section{Conclusion}

The results support the multidisciplinary use of the COPM in clinical practice and research in a home-dwelling, heterogeneous population of older adults. The COPM has adequate content validity, construct validity, and feasibility in this population of older adults and a moderate responsiveness to change. The MIC was established to be 3.0 points and 3.2 points for COPM-P and COPM-S, respectively. The COPM is found to be a useful and manageable instrument in a population of older adults. Test-retest reliability assessments and further responsiveness assessments are needed to supplement the results of this validation study.

\section{Acknowledgments}

We want to thank all the participants and the health care providers who participated in this trial. We also want to thank Bjarte Folkestad, Centre for Care Research Western Norway, for help with the data analysis setup. Finally, we want to thank the Norwegian Directorate of Health who commissioned the study.

\section{Disclosure}

The authors report no conflicts of interest in this work.

\section{References}

1. Law M, Baptiste S, Carswell A, McColl M, Polatajko H, Pollock N. COPM Canadian Occupational Performance Measure (Norwegian Version). 5th ed. Oslo: NKRR National Advisory Unit on Rehabilitation in Rheumatology; 2015.

2. Townsend E, Polatajko H. Enabling Occupation II. Advancing an Occupational Therapy Vision for Health, Well-Being and Justice through Occupation. Canada: Canadian Association of Occupational Therapists; 2007.

3. Langeland E, Førland O, Aas E, et al. Modeller for hverdagsrehabilitering - en følgeevaluering i norske kommuner. Effekter for brukerne og gevinster for kommunene? [In English: Models of Reablement. A Study in Norwegian Municipalities. Effects for Users and Gains for Municipalities?]. Norway: Centre for Care Research Western Norway, Bergen University College, CHARM; 2016.

4. Aspinal F, Glasby J, Rostgaard T, Tuntland H, Westendorp R. Reablement - supporting older people towards independence. Age Ageing. Epub 2016 May 21.

5. Legg L, Gladman J, Drummond A, Davidson A. A systematic review of the evidence on home care reablement services. Clin Rehabil. 2016;30(8):741-749.

6. Tessier A, Beaulieu M-D, MCGinn CA, Lautulippe R. Effectiveness of reablement: a systematic review. Healthc Policy. 2016;11(4):49-59.

7. Kjerstad E, Tuntland H. Reablement in community-dwelling older adults: a cost-effectiveness study analysis alongside a randomized controlled trial. Health Econ Rev. 2015;6(1):15.

8. Tuntland H, Ness NE, editors. Hverdagsrehabilitering [Reablement]. 1st ed. Oslo: Gyldendal Akademisk; 2014. Danish.
9. Tuntland H, Aaslund M, Espehaug B, Førland O, Kjeken I. Reablement in community-dwelling older adults: a randomised controlled trial. $B M C$ Geriatr. 2015;15(146):1-11.

10. Carswell A, McColl MA, Baptiste S, Law M, Polatajko H, Pollock N. The Canadian Occupational Performance Measure: a research and clinical literature review. Can J Occup Ther. 2004;71(4):210-222.

11. Cup EH, Scholte op Reimer WJ, Thijssen MC, van Kuyk-Minis MA. Reliability and validity of the Canadian Occupational Performance Measure in stroke patients. Clin Rehabil. 2003;17(4):402-409.

12. McNulty M, Beplat A. The validity of using the Canadian Occupational Performance Measure with older adults with and without depressive symptoms. Phys Occup Ther Geriatr. 2008;27(1):1-15.

13. Edwards M, Baptiste S, Stratford PW, Law M. Recovery after hip fracture: what can we learn from the Canadian Occupational Performance Measure? Am J Occup Ther. 2007;61(3):335-344.

14. Kjeken I, Slatkowsky-Christensen B, Kvien TK, Uhlig T. Norwegian version of the Canadian Occupational Performance Measure in patients with hand osteoarthritis: validity, responsiveness, and feasibility. Arthritis Rheum. 2004;51(5):709-715.

15. Stuber CJ, Nelson DL. Convergent validity of three occupational selfassessments. Phys Occup Ther Geriatr. 2010;28(1):13-21.

16. Wressle E, Samuelsson K, Henriksson C. Responsiveness of the Swedish version of the Canadian Occupational Performance Measure. Scand $J$ Occup Ther. 1999;6(2):84-89.

17. Kjeken I, Dagfinrud H, Uhlig T, Mowinckel P, Kvien TK, Finset A. Reliability of the Canadian Occupational Performance Measure in patients with ankylosing spondylitis. J Rheumatol. 2005;32(8):1503-1509.

18. Mokkink L, Terwee CB, Patrick DL, et al. The COSMIN study reached international consensus on taxonomy, terminology, and definitions of measurement properties for health-related patient-reported outcomes. J Clin Epidemiol. 2010;63(7):737-745.

19. Eyssen IC, Steultjens MP, Oud TA, Bolt EM, Maasdam A, Dekker J. Responsiveness of the Canadian Occupational Performance Measure. J Rehabil Res Dev. 2011;48(5):517-528.

20. Enemark Larsen A, Carlsson G. Utility of the Canadian Occupational Performance Measure as an admission and outcome measure in interdisciplinary community-based geriatric rehabilitation. Scand J Occup Ther. 2012;19(2):204-213.

21. Nieuwenhuizen MG, de Groot S, Janssen TWJ, van der Maas LCC, Beckerman H. Canadian Occupational Performance Measure performance scale: validity and responsiveness in chronic pain. J Rehabil Res Dev. 2014;51(5):727-746.

22. Langeland E, Tuntland H, Førland O, et al. Study protocol for a multicenter study of reablement in Norway. BMC Geriatr. 2015;15(111):1-9.

23. Jolles BM, Buchbinder R, Beaton DE. A study compared nine patientspecific indices for musculoskeletal disorders. J Clin Epidemiol. 2005;58(8):791-801.

24. Law M, Baptiste S, Carswell A, McColl M, Polatajko H, Pollock N. Canadian Occupational Performance Measure (Norwegian Version). 4th ed. Oslo: National Advisory Unit on Rehabilitation in Rheumatology. Diakonhjemmet Hospital; 2008.

25. Guralnik J, Simonsick E, Ferruci K. A short physical performance battery assessing lower extremity function: association with self-reported disability and prediction of mortality and nursing home admission. $J$ Gerontol. 1994;49(2):M85-M94.

26. Freiberger E, De Vreede P, Shoene D, et al. Performance-based physical function in older community-dwelling persons: a systematic review of instruments. Age Ageing. 2012;41(6):712-721.

27. Haywood KL, Garratt AM, Fitzpatrick R. Quality of life in older people: a structured review of generic self-assessed health instruments. Qual Life Res. 2005;14(7):1651-1668.

28. Antonovsky A. Unraveling the Mystery of Health. San Francisco: Jossey-Bass; 1987.

29. Eriksson M, Lindstrom B. Validity of Antonovsky's sense of coherence scale: a systematic review. J Epidemiol Community Health. 2005;59(6):460-466.

30. Westerhof G, Keyes C. Mental illness and mental health. The two continua model across the lifespan. J Adult Dev. 2010;17(2):110-119. 
31. Lamers SM, Westerhof GJ, Bohlmeijer ET, Ten Klooster PM, Keyes CL. Evaluating the psychometric properties of the mental health continuumshort form (MHC-SF). J Clin Psychol. 2011;67(1):99-110.

32. de Vet H, Terwee C, Mokkink L, Knol D. Measurement in Medicine. Practical Guide to Biostatistics and Epidemiology. Cambridge, UK Cambridge University Press; 2011.

33. Mokkink L, Terwee C, Knol C, et al. The COSMIN checklist for evaluating the methodological quality of studies on measurement properties: a clarification of its content. BMC Med Res Methodol. 2010;10(22): $1-8$.

34. Andresen E. Criteria for assessing the tools of disability outcomes research. Arch Phys Med Rehab. 2000;81(12 Suppl 2):S15-S20.

35. Terwee C, Bot S, De Boer M, van der Windt D, Knol D. Quality criteria were propopsed for measurement properties of health status questionnaires. J Clin Epidemiol. 2007;60(1):34-42.

36. WHO. The International Classification of Function, Disability and Health. Geneva: World Health Organisation; 2001.
37. Harris JE, Eng JJ. Goal priorities identified through client-centred measurement in individuals with chronic stroke. Physiother Can. 2004;56(3):171.

38. Fernandes L, Grotle M, Darre S, Nossum R, Kjeken I. Validity and responsiveness of the measure of activity performance of the hand (MAP-Hand) in patients with hand osteoarthritis. $J$ Rehabil Med. 2012;44(10):869-876.

39. Darzins SW, Imms C, Shileds N, Taylor F. Responsiveness, construct and criterion validity of the personal care-participation assessment and resource tool (PC-PART). Health Qual Life Outcomes. 2015; $13: 125$.

40. Dedding C, Cardol M, Eyssen IC, Dekker J, Beelen A. Validity of the Canadian Occupational Performance Measure: a client-centred outcome measurement. Clin Rehabil. 2004;18(6):660-667.

41. McColl MA, Doubt L, Paterson M, Law M. Validity and community utility of the Canadian Occupational Performance Measure. Can J Occup Ther. 2000;67(1):22-30.
Journal of Multidisciplinary Healthcare

\section{Publish your work in this journal}

The Journal of Multidisciplinary Healthcare is an international, peerreviewed open-access journal that aims to represent and publish research in healthcare areas delivered by practitioners of different disciplines. This includes studies and reviews conducted by multidisciplinary teams as well as research which evaluates the results or conduct of such teams or health

\section{Dovepress}

care processes in general. The journal covers a very wide range of areas and welcomes submissions from practitioners at all levels, from all over the world. The manuscript management system is completely online and includes a very quick and fair peer-review system. Visit http://www.dovepress.com/ testimonials.php to read real quotes from published authors. 